\title{
L'Italie au tournant du siècle : Paradis perdu des homosexuels dans la littérature décadente européenne
}

\author{
Italy at the Turn of the Century: The Lost Paradise of Homosexuals \\ in European Decadent Literature
}

\begin{abstract}
RÉSUMÉ
Révéler l'homosexualité des personnages à travers un voyage en Italie semble un topos caractéristiques des œuvres décadentes au tournant du $\mathrm{XIX}^{\mathrm{e}}-\mathrm{XX}^{\mathrm{e}}$ siècle. La présente étude s'attache aux origines de cette mythologie littéraire à travers une série de textes issus de diverses sources linguistiques en adoptant une méthodologie géocritique. Ce choix d'un pays encore vierge de culture industrielle permet des rencontres épiphaniques dont l'aboutissement est la redécouverte de soi-même par chacun des personnages. Confrontés à des monstres et autres divinités mythologiques secondaires, les protagonistes feront l'expérience de devenir eux-mêmes. Mots-clés : homosexualité, décadence, mythologie, géocritique, italie
\end{abstract}

\section{ABSTRACT}

The choice of Italy to reveal the homosexuality of the characters seems to be a recurring stereotype in the decadent European literature at the turn of the century. The present study examines the origins of such a literary mythology by confronting various linguistic sources in order to identify an intertextual and geocritical network. The choice of a country still untouched by an industrial culture allows for epiphanic encounters whose outcome is the rediscovery of oneself by each of the characters. Confronted with monsters and other secondary mythological deities, the protagonists will experience becoming one themselves.

Keywords: homosexuality, décadence, mythology, géocritical, Italy

Dans son introduction à l'ouvrage La Géocritique mode d'emploi, M. Grassin revient sur la notion d'espace littéraire émergeant en 1955 chez Blanchot pour en proposer la (re)définition suivante : « un lieu réel, matériel, géographique, fantasmé et représenté par la parole» (Westphal, 2000, p. X). Afin d'interpréter cet « espace fantasmé », qu'il soit « [symbolique inspiré ...] par un lieu d'expérience » ou un « [lieu réel parcouru] par les personnages de la fiction », l'universitaire met en

Eric Thil, Lehrstuhl für Allgemeine und Vergleichende Literaturwissenschaft, Fachrichtung Germanistik an der Universität des Saarlandes, A2.2, Campus, 66123, Saarbrücken, thil.eric@yahoo.fr, https://orcid. org/0000-0002-2227-7735 
avant la géocritique qu'il définit provisoirement comme « science des espaces littéraires » seule apte à « interpréter [s]es espaces », en particulier lorsqu'ils sont « imaginaires » (pp. I, XI, XIII).

Si l'Italie est un des pays européens les plus connus de par son histoire, elle est également un terreau fertile pour l'ensemble de la production littéraire occidentale, devenant ainsi, un " espace fantasmé » non seulement d'un seul auteur, mais de plusieurs générations d'auteurs et de mouvements qui ont su en faire un matériau symbolique, voire mythique. En effet, patrie de Rome et donc mère de l'Europe occidentale, c'est à partir du XVIII ${ }^{\mathrm{e}}$ siècle, grâce au père de l'archéologie moderne Winckelmann et avec les « adeptes du 'Grand Tour' » (Hersant, 1988, p. IX), qu'elle connaîtra une nouvelle heure de gloire qui n'a jamais déchu. Or, ces jeunes nobles européens viennent parfaire leur éducation en apprenant non seulement la langue et les arts, mais aussi les us et coutumes, et surtout l'amour dans des bras italiens. En ce sens, il n'est guère étonnant que l'imaginaire romantique en fasse son violon d'Ingres : invitant non seulement à l'évasion mais ouvrant la voie à une postérité littéraire fantasmagorique qui fera de cette nation « une fiction formée de clichés, un pays de pacotille ouvert à toutes les rêveries » (Lund \& Delon, 2008, pp. 6,1$)$.

Cette ouverture sur le possible sans frontière trouvera un écho dans une partie de la littérature fin-de-siècle, que l'on pourrait nommer, faute de mieux, la littérature homosexuelle. Les ouvrages choisis pour ce travail, outre le fait de présenter au lecteur un personnage masculin qui va réaliser le caractère de sa propre nature, possèdent un autre point commun : cette révélation de l'homosexualité se fait au travers d'un voyage en Italie. En effet, au sein de la fiction comme dans la réalité historique, on ne compte plus les exemples de patronymes ayant fui vers ce pays, jugé plus apte à accepter les invertis : comment expliquer ce choix ? L'approche la plus à même d'expliquer ce phénomène ne pouvait donc être autre que géocritique.

Écrits entre 1829 et 1929, les textes de Balzac (Sarrasine, 1829), d'Essebach (Dédé, 1902), de Forster (The Story of a panic, 1911) et de Mann (Tod in Venedig, 1911), tous issus du genre narratif, interrogent le rapport entre la géographie et la sexualité, entre l'espace et la libre personnalité. Cette période de 100 ans est propice à l'avènement de l'homosexualité dans les Lettres, dû en particulier à la montée en puissance des sciences et notamment de la médecine. La physiologie autant que la sexologie investissent les études d'abord réalistes (Balzac), puis naturalistes et enfin décadentes (Essebach, Mann, Forster). Le choix des œuvres fut moins une sélection qu'une évidence imposée au chercheur et qui se justifie par les intertextes découlant d'une œuvre dans l'autre, permettant ainsi l'émergence d'une « chaîne intertextuelle » (Westphal, 2007, p. 193) dont le point d'ancrage/ encrage se trouve dans le Sud de l'Europe.

Le présent examen souhaite dresser un bref panel des choix géographiques dans lesquels les auteurs européens du XIX ${ }^{\mathrm{e}}$ siècle placent les amours de même sexe afin 
d'étudier en quoi ces lieux acquièrent une dimension mythique. Dans un premier temps, il sera bon de dresser un petit inventaire de ces paradis perdus en s'appuyant sur des œuvres de langues française, allemande et anglaise. En établissant des regroupements émergeront ainsi quelques topoï dont il conviendra d'interroger l'origine. Enfin, il s'agira d'étudier la dimension mythique propre à chacun d'eux et le rôle qui en découle pour la pratique esthétique.

\section{A travers ville...}

Des cinq œuvres du corpus, trois choisissent comme décor les zones urbaines italiennes, essentiellement les villes du Nord comme Venise, Vérone ou celles du centre, telle Rome. C'est en effet dans l'actuelle capitale que Sarrasine entreprend d'achever son apprentissage après avoir été l'élève de Bouchardon (se retrouve ainsi le spectre du Grand Tour évoqué en introduction). Ce choix se fait a priori pour des raisons esthétiques : « il vint à Rome, en proie d'inscrire son nom entre les noms de Michel-Ange et de Monsieur Bouchardon » (Balzac, 2011, p. 25). Patrie mythique des Arts, en particulier depuis les travaux de Winckelmann, Rome devient ici le moyen d'évoquer par synecdoque la longue lignée de sculpteurs dont cherche à se revendiquer le protagoniste. Le premier, Michel-Ange, notamment de par ses Sonnets publiés à titre posthume et son travail de sculpture des corps masculins, peut être considéré comme une des figures emblématiques de la culture homosexuelle. Toutefois, Goetz (2003) voit dans le personnage de Balzac un hypertexte d'un article sur Michel-Ange par Delacroix et suggère également mais à mi-mots une possible interaction entre le sculpteur fictif et Vautrin, autre grande figure homosexuelle de La Comédie humaine (pp. 97-98). Qui plus est, il est intéressant de constater que l'ordre choisi par Balzac ne respecte pas la chronologie, Sarrasine désire se placer entre ses deux maîtres masculins, ce qui d'un point de vue artistique revient à montrer que l'élève dépasse le maître même s'il reste en dessous de l'absolu. D'un point de vue psychanalytique en revanche, cela illustre son intérêt pour le même sexe, un intérêt qui l'avait déjà fait renvoyer du collège ${ }^{1}$.

Marcel, le narrateur de Dédé, assume totalement, pour sa part, son amour de collège. Ses sentiments pour André Dalio (le fameux Dédé du titre) sont réitérés à maintes reprises de l'incipit, « je l'aimai », jusqu'à l'excipit, « je t'aime toujours, Dédé » (Essebach, 2005, pp. 9, 236). Ce passage du passé simple au présent permet l'actualisation de cet amour et surtout l'annihilation du trépas,

1 La nouvelle explique le renvoi ainsi : «Enfin, s'il faut en croire la chronique du collège, il fut chassé, pour avoir, en attendant son tour au confessionnal, un vendredi saint, sculpté une grosse bûche en forme de Christ. L'impiété gravée sur cette statue était trop forte pour ne pas attirer un châtiment à l'artiste » (Balzac, 2011, pp. 23-24). La métaphore de l'impiété gravée pourrait être justement d'ordre sexuel... 
phénomène redoublé par la répétition du verbe aimer tout au long du récit et qui prend dès lors une valeur incantatoire. Ce dépassement de la mort se fait après que le narrateur a visité le caveau familial où son jeune ami est enterré des suites d'une tuberculose. Il se situe alors à Vérone, ville shakespearienne emblématique pour des amants. Cependant ce n'est plus auprès d'une Juliette décédée que vient se lamenter Roméo ; c'est auprès d'un autre Roméo! Amusante ironie à la pensée que Shakespeare, tout comme Michel-Ange, fut l'auteur de sonnets à dominance homo-érotique... La saison automnale favorise la mélancolie du personnage qui, en héros à l'héritage romantique, se languit et se lamente de la perte de son heureux passé et de cette jeunesse qui s'en est allée avec la mort de Dédé. Cependant cette élégie sera l'occasion pour lui de rencontrer un " gentil compagnon » (Essebach, 2005, pp. 216-218) venu prier dans l'Eglise mitoyenne. Ce contact sera pour lui le moyen d'accomplir (en partie) son deuil, se laissant troubler par la beauté et la voix de l'adolescent « première étoile [parue] dans le ciel » depuis la disparition de son camarade.

Le décès accepté, c'est à Venise qu'émigre Marcel durant cette même saison automnale. Il y rencontre un gondolier, Andrea, qui le conduit bien et tente de le séduire. Le soir même, il prend une autre gondole et se fait chanter un De profundis par des musiciens connus du batelier. Ironie du sort, l'un des musiciens porte également le nom d'Andrea et son physique évoquera une nouvelle fois son ami enterré. Ce personnage ajouté à la musique plongera le narrateur dans un délire qui lui permettra d'accomplir véritablement son deuil, puisqu'il croit voir et entendre « les morts » (Essebach, 2005, pp. 237-238). À la différence de Marcel, le voyage d'Aschenbach dans le récit de Thomas Mann le conduit bien à Venise ${ }^{2}$, mais pour y connaître lui-même sa fin, mourant du choléra en contemplant Tadzio (Mann, 2007, p. 139). Venise est donc synonyme de beauté mais aussi de mort pour le romancier allemand, ce qui peut être un hommage à Barrès dont le texte La Mort de Venise est publié en 1903, soit un an après Dédé.

\section{Et campagne}

Seuls les personnages Forster s'octroient des vacances à la campagne. La famille anglaise Tytler, c'est dans la campagne de Ravello, à proximité de Pompéi, qu'elle passe ses vacances, endroit qualifié d' " endroit charmant avec un charmant petit hôtel dans lequel nous [les Tytler] avons rencontré des gens charmants » (Forster, 1948, p. 1). Malgré une dimension proprement arcadienne à l'endroit, la répétition de l'adjectif qualificatif et la chute de la phrase implique l'ironie du narrateur homodiégétique. La rencontre des touristes avec le dieu Pan aura également lieu en

2 Après un arrêt à Trieste où le narrateur ne se sent pas à l'aise... probablement parce que c'est dans cette ville que Winckelmann a été assassiné en $1768 \ldots$ En ce qui concerne les affinités d'Aschenbach avec Winckelmann, consulter l'ouvrage de Dolberg. 
pleine nature, plus précisément dans une vallée ayant l'aspect d'une coupe, voire d'une paume entourée par des monts qui en forment les doigts. Si la coupe pourrait à priori rappeler l'ivresse de Dionysos (dont le cortège est souvent accompagné de satyres tels que Pan), la personnification du décor en main rappelle que, dans les contrées sauvages, les dieux ne sont pas morts et que les humains sont encore entre les mains des divinités, en l'occurrence, celle de Pan, ainsi que le prouvent les traces de chèvre (pp. 3-11) ainsi que la figura etymologica du titre ${ }^{3}$.

\section{Synthèse}

Une opposition traditionnelle entre ville et campagne figure donc au sein des œuvres de fictions. Pourtant, cette opposition s'avère factice, puisque chacune d'elles fait osciller les personnages entre le monde sauvage et celui de la civilisation. Le retour en Italie symbolise un retour à l'état sauvage, à l'état de nature, hors de la réalité positiviste qu'était celle des lecteurs du XIX siècle. Dans quatre cas, en parallèle des figures qui visitent un endroit étranger, le texte bascule dans une sorte d'inquiétante étrangeté : ainsi la panique irréfléchie des adultes chez Forster (provoquée par le dieu Pan) pourrait se lire comme la peur irraisonnée devant une possible homosexualité. Seul témoin de cette rencontre épiphanique, Eustace en sortira changé : d'une part il se rapproche de la nature et reconnaît sa poésie (Forster, 1948, p. 21) et d'autre part il se rapproche physiquement et intellectuellement de Gennaro ${ }^{4}$. Se pourrait-il que le dieu ait connu charnellement l'adolescent ? C'est à cette conclusion qu'invite Forster lorsqu'il déclare qu'au moment de se lever pour rentrer, «Eustace marcha avec difficulté, presque avec douleur » (p. 12), invitant à une possible lecture intertextuelle avec l'œuvre d'Arthur Machen The Great God Pan (1894). Pour Aldrich (2002), «The Story of a Panic [montre] le pouvoir continu de l'Italie de réaliser la révélation sexuelle (qu'elle soit hétérosexuelle ou homosexuelle) »(p. 100), sexualité considérée comme taboue et qui ne pouvait ni être énoncée clairement ni vécue officiellement dans l'Angleterre victorienne.

À bien des égards, la 'transformation' (pour ne pas parler de révélation) de Zambinella en 'homme' peut se lire comme un événement étrange et n'a lieu qu'après le passage à Frascati. Il est intéressant de noter que le personnage masculin n'entend pas le double discours du castrat : il ne relève par exemple pas l'emploi du masculin ou refuse d'envisager l'innommable «-Si je n'étais pas une femme ? [...]-La bonne plaisanterie ! (Balzac, 2011, pp. 36-37). Sarrasine reste sous l'effet de la voix de cette

3 Le substantif panic provenant à la base de l'adjectif grec panikós, (une terreur provoquée par le dieu Pan). À cause de l'antonomase, le vocable existe aujourd'hui dans les deux états et ce dans de nombreuses langues (ainsi en français, en anglais, en allemand, etc.).

4 C'est également l'hypothèse formulée par Hammond (1996, p. 196). Sauf en ce qui concerne la relation intime, Merivale (1969, pp.180-193) arrive aux mêmes conclusions. 
sirène moderne... Un serpent se dresse sur le passage de Zambinella, ce qui rassure Sarrasine qui voit dans la peur de l'autre la preuve de son caractère féminin. Au-delà du clin d'œil à la Genèse, le serpent semble un avertissement du morceau manquant de Zambinella. Bien qu'il ne s'agisse que d'une couleuvre, l'imaginaire collectif prêté à cet animal, qui en fait un suppôt du diable et des sorcières, lui attribue généralement de noirs desseins. D'un point de vue psychanalytique, il devient alors la métaphore même de Zambinella, créature punie par la divinité pour avoir trompé.

En ce qui concerne les gondoliers d'Essebach, ils représentent Charon le passeur de l'autre monde, seul apte à établir un lien entre la mémoire du mort et le souvenir du vivant. En prenant l'identité (la voix, le chant) du disparu Dédé, ces bateliers se transforment également en des figures d'Orphée, mais subvertissent le mythe puisque c'est de l'au-delà que provient le chant qui attire le narrateur Marcel, comme les sirènes vers les récifs. À la fin du siècle, Orphée ne déplore plus la perte de son Eurydice, mais celle de son compagnon ${ }^{5} .$. Aschenbach, lui aussi, fera une expérience similaire en embarquant sur une gondole dont le conducteur disparaîtra mystérieusement après avoir prononcé la fameuse sentence proleptique « Vous payerez » (Mann, 2007, pp. 45-46). Armand Nivelle propose de voir dans les diverses figures qui ornent le passage de l'écrivain, Hermès psychopompe, qui en plus de mener Aschenbach de l'ordre vers le chaos, de la beauté vers la sensualité (ou d'Apollon vers Dionysos), le mènera à sa propre perte. Là où un Essebach se voyait encore optimiste concernant les relations de même sexe, Thomas Mann se voit plus pessimiste en démontrant les effets dévastateurs de la passion homosexuelle.

À croire que le fantastique a partie liée avec l'homosexualité, ainsi que le rappelle Tzvetan Todorov, paraphrasant Penzoldt : "'le surnaturel n'est qu'un prétexte' » permettant ainsi « de franchir certaines limites inaccessibles » du discours et de contourner par ce biais la « censure institutionnalisée » (Todorov, 1970, pp. 166-167). C'est bien l'étrangeté présente au sein des textes qui amène la révélation de l'homosexualité. Or celle-ci ne peut avoir lieu que dans un cadre étranger, exotique. Quoi de plus approprié que l'Italie, patrie romaine, où le « vice italien » (Aldrich, 2002, p. 34) remplaça à partir de la Renaissance l'amour grec. La présence d'une importante forme de religiosité retarde l'arrivée des Lumières et de l'urbanisation telle que la connaissent les pays en pleine révolution industrielle. Or, la sexualité représente la part bestiale de l'homme dans ces mêmes pays, ce retour en Italie représente donc bien une métaphore d'un retour à la nature sauvage, aux origines et donc au plaisir sexuel, mais peut être pour le même coup considéré comme l'élément déclencheur de la monstruosité.

\footnotetext{
5 À noter que certaines versions du mythe suggèrent que ce dernier, après la mort de sa jeune épouse et son échec aux Enfers, se tourne vers les hommes. Pour davantage d'informations, consulter l'article de Brisson (2012).
} 


\section{Dans la grotte où nage la sirène...}

Qui dit lieu étrange, dit souvent monstre. Ce phénomène se voit amplifié par la réalité côtière de l'Italie que la mer Méditerranée entoure ; l'eau, frontière poreuse, permettant l'accès à l'autre monde depuis l'Antiquité (avec la figure de Charon notamment). Or, ce fameux Grand Tour des divers personnages ne pourrait-il pas se lire comme une forme atténuée de l'Odyssée, voyage durant lequel les protagonistes vivent d'étonnantes aventures qui les reconduiront chez eux ? C'est alors en ce sens qu'il faut comprendre la métaphore des sirènes, implicitement développée par la voix chantée tant dans Sarrasine que dans Dédé.

Chez Balzac (2011, pp. 27-28), la métaphore filée de l'élément aérien pour désigner ce timbre enchanteur déchaîne littéralement le sculpteur qui s'oublie en société et fait partager son extase à toute la salle. La sirène Zambinella ${ }^{6}$ a quitté son île pour s'afficher à l'opéra et déclenche de par son chant la passion de Sarrasine, ce qui l'attire irrémédiablement vers elle. À noter toutefois que le narrateur connaît la nature de Zambinella, usant de l'adjectif diabolique comme d'une prolepse et jouant sur l'étymologie du substantif diable qui signifie trompeur. Car si ce sont finalement les amis de Zambinella qui veulent jouer une farce à Sarrasine, c'est bien sa voix qui en perfectionne l'artifice, jugée tour à tour empreinte de faiblesse, d'ange, céleste; la faute du castrat est donc de tromper par sa voix. Cette gradation vers les hautes sphères est inversement proportionnelle à la chute du sculpteur qui vit après la révélation un véritable enfer, à en juger par son rire « infernal» (pp. 37, 39, 40, 41). La sirène, une fois sur la scène de théâtre, ne mène plus le personnage à la simple mort, mais véritablement à l'enfer, qui était le sort réservé aux sodomites et invertis durant des siècles.

Ce supplice d'être sous le charme d'une voix tout en réalisant l'impossibilité d'une relation, Marcel, le narrateur de Dédé en fait également l'expérience à bord de la gondole du batelier Andrea dont le chant va jusqu'à la torture (Essebach, 2005, p. 228). Cette voix de l'entre deux peut aisément se rapprocher de celle de Zambinella ${ }^{7}$ (ou d'un castrat quelconque). Là encore, le chant provoque la frustration et la douleur du narrateur, et va jusqu'à contaminer la lagune qu'elle incendie. La sirène, ici masculine, se confond avec Orphée et ramène à la vie le chanteur disparu en substituant le rêve à la réalité. À l'inverse d'Aschenbach qui vit également une descente aux enfers, le narrateur d'Essebach se voit transfiguré

${ }^{6}$ Il est intéressant de voir que la voix de Zambinella transcende les générations de manière héréditaire ; ainsi la famille du castrat, les Lanty, ont une fille, Marianina, à la « voix enchanteresse ». Quant à sa mère, la nièce même du castrat, elle est comparée à l'une des " puissantes sirènes » (Balzac, 2011, pp. 10-11).

7 Zambinella dont l'un des modèles possibles (sinon l'archétype) pourrait être Farinelli, ainsi que le suggère Barthes (1984, p. 178). À noter qu'être castrat implique un refus de laisser muer la voix et donc de laisser grandir la personne, ce qui, d'un point de vue psychanalytique devient une forme détournée de pédérastie... 
par Venise et sa beauté. Cet assemblage des contraires (Bianchi, 2018, p. 14) ne peut se faire qu'à Venise, seule ville italienne située à la fois sur terre et sur la mer (p. 98), véritable hétérotopie au sens où l'entendait Foucault ${ }^{8}$.

\section{Dans le labyrinthe}

Cette hétérotopie permet également de superposer différents mythes, à la manière d'un palimpseste, ce qui explique alors que trois des textes du corpus présentent la ville italienne comme un labyrinthe. En effet, après avoir vu se produire Zambinella, Sarrasine est contacté par une duègne qui l'emmène jusqu'au castrat à travers un labyrinthe d'escaliers, de galeries et d'appartements (Balzac, 2011, p. 31). L'atmosphère lugubre et aventureuse conduit le protagoniste jusqu'au cœur du labyrinthe où il rencontrera le castrat, et sa perte. Contrairement à un Thésée héros de son temps, Sarrasine n'est pas maître de lui-même mais subit sa passion. Même constat pour Marcel (Essebach, 2005, p. 221) et Aschenbach (Mann, 2007, p. 131) qui se perdent tous deux dans les ruelles de Venise. Si le premier erre simplement, le second oriente sa recherche vers un objet précis, le jeune Tadzio. Cependant, Venise ne fait que les confronter à des impasses, autrement dit à leur propre solitude. Ce renvoi à soi correspond à la quête identitaire que les personnages recherchent à Venise (Bianchi, 2018, p. 14).

Comme le rappelle Bertrand Westphal (2007, p. 13), « chaque labyrinthe spatialement hiérarchisé dispose d'un monstre en son centre ». Or le monstre, assemblage de plusieurs espèces différentes, est ce que trouve Sarrasine à Rome, et dont l'attirance fait également de ce dernier une forme de monstre : « tu m'as ravalé jusqu'à toi » (Balzac, 2011, p. 42). En plus de la connotation cannibalesque (un rappel de plus pour les sirènes), le verbe implique un abaissement, une transposition de Zambinella en Sarrasine. En réalisant que tout est vide de sens pour lui comme pour le castrat, le sculpteur devient un monstre à son tour, homme qui n'en est pas un parce qu'il a aimé l'image d'une femme présente dans un homme privé de sa virilité. C'est donc bien la révélation de l'homosexualité qui entraîne la transformation des figures romanesques. Ainsi Eustace, après son expérience épiphanique agira comme une chèvre (Forster, 1948, p. 15) durant le temps de ce qui peut être désigné comme une initiation (dont la finalité sera sa transfiguration comme artiste). Dans le cas d'Aschenbach, le changement est plus subtil, puisque la contemplation de Tadzio lui communiquera l'envie de plaire en s'offrant une seconde jeunesse. Néanmoins, il prendra l'apparence d'une autre figure qu'il avait déjà rencontrée lors de son voyage vers Trieste, le vieux beau. Car les artifices qu'exécrait Aschenbach chez cet imposteur (Mann, 2007, p. 34), l'écrivain vieillissant par l'intermédiaire de son coiffeur les utilisera aussi

8 Concernant Venise et l'hétérotopie, consulter les ouvrages de Westphal (2000, p. 24) et Bianchi (2018, p. 68). 
(pp. 128-131), acceptant par là sa révélation : sa passion inconditionnelle pour un jeune Polonais, autrement dit sa pédérastie ${ }^{9}$. Cette fin qui peut se lire soit comme un châtiment divin, soit comme une transfiguration de l'artiste est aussi ambiguë que le pays dans lequel elle se déroule...

\section{Vers une mythologie homosexuelle}

L'Italie a attiré au cours des siècles de nombreux homosexuels. Selon Aldrich (2002, p. 57), c'est parce qu'elle se trouvait être « un endroit où fuir les inhibitions et où trouver de la culture ». La culture dont il est question est notamment liée à l'esthétique, mise en évidence par Winckelmann vers le milieu du XVIII ${ }^{\mathrm{e}}$ siècle. La littérature homosexuelle, en transposant son univers diégétique en Italie, rend alors hommage au père de l'archéologie moderne (Darriulat, 2007). Analysant la réception du personnage historique dans la fiction allemande du $\mathrm{XX}^{\mathrm{e}}$ siècle, Dolberg (1976) indique la propension des auteurs à participer à la mise en place d'un mythe littéraire (pp. 13-14). Ainsi, la biographie de l'archéologue devient le palimpseste sur lequel les histoires d'artistes en quête de beauté seront narrées, de leur début jusqu'à leur perte, instaurant un jeu de références intertextuelles. Le voyage et la mort de Winckelmann pour l'Italie sont à la base des textes de Balzac et de Mann (pp. 102-103). Son intérêt pour la culture antique et pour la beauté permettra de former le caractère de Marcel.

Le second point où se rejoignent les œuvres est l'unanime accord de montrer que le voyage vers le Sud, en particulier l'Italie, est à lire comme la métaphore d'une renaissance de la vigueur sexuelle. Ce n'est donc pas un hasard que Marcel désire s'y rendre après avoir fait la connaissance d'un personnage qui bouleverse ses sentiments. Une évolution sera néanmoins sensible à partir de l'apparition du mouvement connu sous le nom de décadence : les dandys essoufflés par la fin du siècle (dont ils sont la personnification) feront une cure en Italie dans le but de retrouver une sensualité (et une sexualité) inexistante chez eux. Néanmoins, en bons dilettantes, ils se révéleront à eux-mêmes, à l'instar du jeune Eustace chez Forster, ou encore du respectable écrivain chez Mann.

Une dernière référence sera le passage obligé par Venise. « Venise n'est sans doute pas qu'un endroit mythique ; plus globalement, elle constitue peut-être la métaphore idéale du Mythe », soutient Westphal (2000, p. 13). Si cette cité est vue comme une sorte de garde-fou capable de soigner les maux dont souffrent les décadents, à l'instar du personnage éponyme d'un roman de Lorrain, Monsieur

9 Malgré la différence d'âge, pédérastie est préférable dans le sens où Thomas Mann, par les nombreuses références intertextuelles à la philosophie grecque, fait de ses deux protagonistes Eraste et Eromène, mais dont l'initiation est d'abord inversée (c'est au jeune homme de donner une leçon au vieillard) puis ratée (le départ de l'un entraîne la mort de l'autre, alors que dans la civilisation grecque, la séparation impliquait l'évolution du plus jeune). Pour plus de détails, consulter l'ouvrage de Bernard Sergent. 
de Phocas, c'est après l'irrévocable titre de Barrès en 1903 que la ville deviendra la ville de l'inavouable (Basch, 2000, p. 167), expliquant ainsi le bouleversement s'opérant entre un Essebach qui y célèbre l'homosexualité de manière lyrique et un Mann qui l'ironise sur le mode tragique. Cette cité labyrinthique (Basch, 2000, p. 38), parce qu'elle est malade (Bianchi, 2018, p. 123), " agit comme une force négative, une puissance du mal » (Basch, 2000, p. 167) qui va perdre les personnages en leur révélant leur propre monstruosité. La ville devient ainsi un miroir des perversions de tout un imaginaire fin-de-siècle.

\section{Conclusion}

Il est vrai que ledit imaginaire avait été préparé par les ruines d'un Romantisme issu droit des œuvres de Mme de Staël ou de Stendhal. L'Italie est un pays privilégié quant à la cristallisation du sentiment amoureux, qu'il soit hétéro- ou homosexuel d'ailleurs. La chaleur et l'hospitalité du Sud est programmatique du réveil des sens et des sentiments, cela est un fait, mais pourquoi avoir favorisé ce lieu, alors même qu'il se trouvait être le refuge des papes (Rome) et d'une police inquisitrice (Venise) des plus intransigeantes ? Paradoxalement, pour cette même raison. Les Lumières n'ayant pas encore complètement illuminé ce territoire, la culture y restait inférieure à la nature. Or, si c'est bien la nature qu'exécraient les décadents, ces derniers ne résistaient pas à l'appel de l'exotisme, exotisme qui plus est tourné vers la beauté et le passé dans le cas de l'Italie. C'est bien de cette idéologie que la littérature décadente tira profit. Les représentants de la littérature homosexuelle, en particulier, subvertirent ce topos de l'amour romantique pour en faire un mythe 'populaire', conformément à la définition qu'en donne Barthes dans son ouvrage Mythologies. C'est néanmoins avec les guerres du $\mathrm{XX}^{\mathrm{e}}$ siècle que cesseront ces voyages en direction du paradis perdu.

\section{References}

Aldrich, R. (2002). The Seduction of the Mediterranean. London: Taylor \& Francis e-Library.

Barthes, R. (1984). Masculin, féminin, neutre. Le Genre humain, 10, 171-187. Retrieved April 11, 2020, from https://www.cairn.info/revue-le-genre-humain-1984-1-page-171.htm.

Basch, S. (2000). Paris-Venise 1887-1932. Paris: Champion.

Bianchi, A. (2018). Venedig als Labyrinth. Regensburg: Schnell \& Steiner.

Brisson, L. (2012). Orphée, le chant de l'immortalité. Le Point références, A4, pp. 82-83.

Darriulat, J. (2007). Balzac "Sarrasine" (1830), Retrieved April, 11, 2020, from www.jdarriulat.net/ Auteurs/Balzac.html.

De Balzac, H. (2011/1829). Sarrasine. In E. Richter, K. Struve, \& N. Ueckmann, Balzacs Sarrasine und die Literaturtheorie. Zwölf Modellanalysen (pp. 7-44). Stuttgart: Reclam.

Dolberg, G. A. (1976). The Reception of Johann Joachim Winckelmann in modern german prose fiction. Stuttgart: Hans-Dieter Heinz.

Essebach, A. (2005). Dédé. Paris: Quintes-Feuilles.

Forster, E. M., (1948/1911)). The Story of a Panic. In Collected Shortstories of E. M. Forster (pp. 1-29). London: Sidgwick \& Jackson. 
Goetz, A. (2003). L'esthétique italienne dans „C“Comédie humaine“. In Balzac et l'Itali, Actes de colloque international, Maison de Balzac-institut culturel italien 2000 (pp. 88-103). ParisMusées: Éditions des Cendres.

Gusdorf, G. (1948). La Découverte de soi. Paris: Presses universitaires de France.

Hammond, P. (1996). Love between men in English literature. London: Macmillian Press.

Hersant, Y. (1988). Italies. Paris: Robert Laffont.

Lund, H.-P., \& Delon, M. (Eds.). (2008). L'Italie dans l'imaginaire romantique. Copenhagen: Det Kongelige Danske videnskabernes selskab.

Mann, T. (2007/1911). Der Tod in Venedig. Stuttgart: Fischer Verlag.

Merivale, P. (1969). Pan the goat-god. Cambridge: Harvard University Press.

Sergent, B. (1984). L'Homosexualité dans la mythologie grecque. Paris: Payot.

Todorov, T. (1970). Introduction à la littérature fantastique. Paris: Seuil.

Westphal, B. (Ed.). (2000). La Géocritique mode d'emploi. Limoges: Presses Universitaires de Limoges.

Westphal, B. (2007). La Géocritique. Rréel, fiction, espace. Paris: Minuit. 